\title{
Implementation of projective measurements with linear optics and continuous photon counting
}

\author{
Masahiro Takeoka and Masahide Sasaki \\ Quantum Information Technology Group, \\ National Institute of Information and Communications Technology (NICT), \\ 4-2-1 Nukui-kitamachi, Koganei, Tokyo 184-8795, Japan and \\ CREST, Japan Science and Technology Agency, 1-9-9 Yaesu, Chuoh-ku, Tokyo 103-0028, Japan \\ Peter van Loock \\ Quantum Information Science Group, National Institute of Informatics (NII), \\ 2-1-2 Hitotsubashi, Chiyoda, Tokyo 101-8430, Japan \\ Norbert Lütkenhaus \\ Quantum Information Theory Group, Zentrum für Moderne Optik, \\ Universität Erlangen-Nürnberg, 91058 Erlangen, Germany
}

(Dated: September 6, 2018)

\begin{abstract}
We investigate the possibility of implementing a given projection measurement using linear optics and arbitrarily fast feedforward based on the continuous detection of photons. In particular, we systematically derive the so-called Dolinar scheme that achieves the minimum error discrimination of binary coherent states. Moreover, we show that the Dolinar-type approach can also be applied to projection measurements in the regime of photonic-qubit signals. Our results demonstrate that for implementing a projection measurement with linear optics, in principle, unit success probability may be approached even without the use of expensive entangled auxiliary states, as they are needed in all known (near-)deterministic linear-optics proposals.

PACS numbers: 03.67.Hk, 03.65.Ta, 42.50.Dv
\end{abstract}

\section{INTRODUCTION}

The implementation of positive operator-valued measures (POVMs) for photonic quantum state signals is important for a variety of quantum information protocols, in particular, for quantum communication schemes such as quantum teleportation [1], quantum key distribution 2], and collective decoding in quantum channel coding $3,4,5$. 5. Unlike conventional optical detection technologies, POVMs for optical quantum information protocols generally include a projection onto superposition states or entangled states. In order to implement such measurements, normally a nonlinear interaction of the signal states (described by a Hamiltonian at least cubic in the optical mode operators [ 6]) is needed. At present, however, these nonlinear processes are hard to realize on the level of single photons.

One possibility for inducing a nonlinear element is to exploit the effective nonlinearity associated with a measurement. In particular, for photonic-qubit states, universal gating operations and hence any POVM for these states can be realized asymptotically by using linear optics, photon counting, highly entangled auxiliary states of $n$ photons, and conditional dynamics (feedforward). Here, conditional dynamics means the successive application of linear transformations on the remaining modes conditioned upon the detection of a subset of modes [7]. In the special case of a projection measurement, corresponding to the discrimination of an orthogonal set of states, perfect distinguishability is achieved in the asymptotic limit of large $n$. However, with current technology, it is hard to generate the entangled auxiliary states even for modest $n$. More recent investigations, therefore, have focused on the question whether one can implement a given measurement, or more generally a set of universal quantum gates, via cheaper and/or finite resources. For example, by applying the clusterstate model of quantum computation [8] to linear optics [9], the cost of the extra entangled resources may be significantly reduced [9, 10].

In this paper, we address the following question: is it possible to implement a given projection measurement in the asymptotic limit of infinitely many, arbitrarily fast conditional-dynamics steps without using any entangled auxiliary states at all? Thereby, the intermediate detections upon which the conditional dynamics relies are not supposed to be finite either, but they shall be arbitrarily weak. In other words, instead of using arbitrarily expensive auxiliary states of arbitrarily many photons and a finite number of finite measurements plus feedforward [7], we employ infinitely many steps of feedforward alone, based upon arbitrarily weak measurements. In fact, a nice example for the latter approach was already given by Dolinar [11] in the field of quantum communication and detection theory, namely, for the minimum error discrimination of binary coherent signals.

As an extension of the conventional signal detection theory, quantum detection theory has been studied to give an optimal signal decision strategy for noncommutative quantum signals [12]. It is motivated by fundamental 
interest, but it also aims at investigating ultimate performance of optical communication systems where information is usually carried by coherent-state signals. Now, in this context, the simplest scenario would be a communication scheme that is based on the discrimination of the binary phase-shift keyed (BPSK) coherent signals $\{|\alpha\rangle,|-\alpha\rangle\}$ (in the following, simply called the binary coherent signals). The optimal POVM that discriminates these nonorthogonal signal states with the minimum average error is described by a projection onto the orthonormal basis consisting of superposition states of $|\alpha\rangle$ and $|-\alpha\rangle$. This scheme is sometimes called the Helstrom measurement [12]. Kennedy 13] first showed a simple physical model that achieves the near-optimal measurement: the binary signal is displaced to $\{|2 \alpha\rangle,|0\rangle\}$ and then measured by a photodetector that discriminates whether the signal contains photons or not. Dolinar [1] (see also 12]) extended the Kennedy scheme demonstrating that the perfect implementation of the Helstrom measurement is possible by using linear optics, photon counting, and infinitesimally fast feedforward. Other related proposals on the implementation of the (near-)optimal measurement of binary coherent states were given in Refs. 14, 15, 16].

A brief description of Dolinar's original proposal is the following 11]. As shown in Fig. 1] the coherent signal field $s_{i}(t)(i=0,1)$ within the time interval $T$ is displaced by one of the two local oscillators (LOs) $\beta_{0}(t)$ and $\beta_{1}(t)$ and then incident into a photodetector. The photodetector is assumed to have infinitesimal time resolution and the functions $\beta_{0}(t)$ and $\beta_{1}(t)$ are appropriately chosen to minimize the error probability. The choice of $\beta_{0}(t)$ or $\beta_{1}(t)$ at time $t^{\prime}\left(0 \leq t^{\prime}<T\right)$ depends on whether the totally detected number of photons during $\left[0, t^{\prime}\right)$ is even or odd. Therefore, once a signal photon triggers a detector click, the current LO immediately has to be switched to the other setting. After detecting the whole signal, one can infer whether the signal was $s_{0}(t)$ or $s_{1}(t)$ by looking at the parity of the number of the detected photons. This scheme achieves the minimum error probability after making an optimization of the likelyhood ratio of the binary signal with optimal control theory [11, 17]. In the original paper [11], the analysis was semiclassical and later a fully quantum mechanical description was given 18]. More recently, the system performance under the realistic situation including delay of feedforward, finite bandwidth and imperfect detection has been investigated [17, 19].

Compared to previous works on the Dolinar scheme, our contribution here contains basically four new aspects. First, we revisit the derivation of a Dolinar-type measurement scheme. However, instead of focusing on the original signals $\{|\alpha\rangle,|-\alpha\rangle\}$, we simply consider the projection measurement onto the basis $\left\{\left|\omega_{0}\right\rangle,\left|\omega_{1}\right\rangle\right\}$ that corresponds to the minimum error discrimination of the signal states. We can then ask whether one can discriminate the orthogonal states $\left\{\left|\omega_{0}\right\rangle,\left|\omega_{1}\right\rangle\right\}$ via linear optics and infinitely weak detections. In such a scheme, during the entire measurement, the conditional states in every

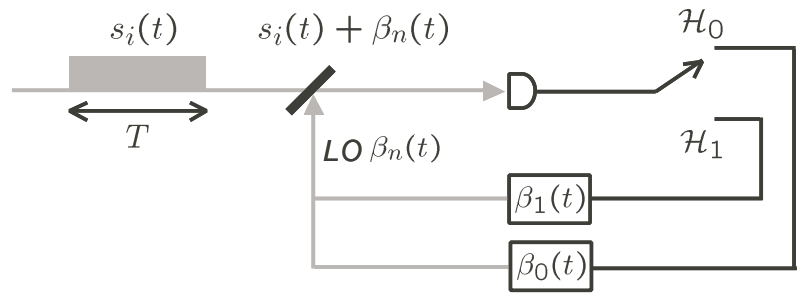

FIG. 1: The Dolinar receiver. The signal $s_{i}(t)$ and the local oscillator are combined at a highly transmissive beamsplitter such that the signal is displaced to $s_{i}(t)+\beta_{n}(t)$. The appropriate choice of $\beta_{n}(t)$ at $t^{\prime}$ is determined by the number of the detected photons during $\left[0, t^{\prime}\right)$ (For details, see the text).

detection and feedforward step must remain orthogonal. Using this constraint, the derivation of the Dolinar protocol becomes simpler and more transparent. Further, no complicated optimization procedures are needed. In order to discuss the Dolinar receiver in the context of linear-optics quantum information processing, we translate Dolinar's original scheme from the time domain to the spatial domain. In other words, infinitesimally fast feedforward is replaced by an infinite use of spatial resources.

Secondly, we prove that there is no finite detection scheme that attains the Helstrom bound for the minimum error discrimination of binary coherent states. Such a nogo statement can be made by using a set of criteria for the exact discrimination of orthogonal states in a projection measurement [20]. These criteria express in a simple way the requirement that the orthogonal states must remain orthogonal after a linear-optics transformation followed by the detection of a first mode. For instance, in a Bell measurement for polarization-encoded photonic qubits, this requirement can never be met and hence the Bell measurement cannot be implemented with linear optics including photon counting, finite steps of conditional dynamics, and arbitrary auxiliary photon states [20, 21, 22]. Asymptotic schemes 7] are not included in this approach. For the example of binary coherent states investigated here, finite feedforward means that the linear-optics transformation of the signal and auxiliary modes before the first detection step involves a nonzero mixing between the signal mode and the mode to be detected. Of course, in the case of zero mixing, the orthogonality can be trivially preserved. Here we will show how to accomplish the Helstrom measurement based on feedforward using infinitely weak detections.

A third new aspect of our work is to demonstrate that the Dolinar approach can be applied not only to the Helstrom measurement of binary coherent states, but also to other measurements. In particular, we apply the Dolinar approach to a projection measurement in the regime of photonic-qubit signals. For a particular example, for which any finite linear-optics scheme must fail, we show that the Dolinar approach succeeds.

Last but not least, our analysis of the Dolinar scheme 
in the view of the recent developments in linear-optics quantum information processing demonstrates that by using infinitely many steps of feedforward instead of arbitrarily expensive entangled photon states, an asymptotically perfect efficiency of a projection measurement is possible. Related to this, we note that there is another proposal for an asymptotic linear-optics implementation of a quantum measurement in which suboptimal unambiguous state discrimination (USD) of $N$ symmetric coherent states for $N \geq 3$ is achieved without expensive auxiliary resources [23]. For $N=2$, the optimal USD can be easily done non-asymptotically by using a $50 / 50$ beamsplitter and photon counting [24].

\section{CONTINUOUS MEASUREMENT VIA LINEAR OPTICS AND CONDITIONAL DISPLACEMENTS}

In this section, we describe a linear-optics circuit in which a sequence of arbitrarily weak measurements are asymptotically combined to a continuous measurement. In particular, we discuss the linear-optics circuit that corresponds to the Dolinar receiver in the spatial domain. A fully quantum-mechanical description of the Dolinar receiver was described in Ref. [18] by applying a continuous photon-counting measurement based on the quantum Markov process model [25, 26].

In the following, we describe the continuous measurement based on photon counting with a local oscillator by a sequence of linear optics and photodetection. The equivalence between continuous photon counting process and a sequence of beamsplitters and photodetectors was firstly pointed out in Ref. [27]. A schematic of our measurement model is shown in Fig. 2(b). The scheme consists of a sequence of weak photodetection steps. In each measurement step, a small fraction of the signal state is reflected by a beamsplitter and measured by a photodetector after a displacement operation [Fig. 2(a)]. The photodetector counts the photon number of the signal.

After one measurement step, the output state conditioned upon the number of detected photons $k$ is described by

$$
\hat{\rho}_{\text {out }}^{(k)}=\frac{\operatorname{Tr}_{B}\left[\left(\hat{I}^{A} \otimes \hat{\Pi}_{k}^{B}\right) \hat{\rho}_{\text {out }}^{\prime}\right]}{\operatorname{Tr}_{A, B}\left[\left(\hat{I}^{A} \otimes \hat{\Pi}_{k}^{B}\right) \hat{\rho}_{\text {out }}^{\prime}\right]},
$$

where

$$
\begin{aligned}
\hat{\rho}_{\mathrm{out}}^{\prime}= & \hat{D}^{B}(\beta \sin \theta) \hat{B}^{A B}(\theta)\left(\hat{\rho}_{\text {in }}^{A} \otimes\left|0^{B}\right\rangle\left\langle 0^{B}\right|\right) \\
& \times \hat{B}^{\dagger A B}(\theta) \hat{D}^{\dagger B}(\beta \sin \theta),
\end{aligned}
$$

and $\hat{\rho}_{\text {in }}$ is the input state. $\operatorname{Tr}_{A}, \operatorname{Tr}_{A, B}$ denote the trace operations over the mode $A$ and the modes $A$ and $B$, respectively. $\hat{B}^{A B}(\phi)=\exp \left[\phi\left(\hat{a}^{\dagger} \hat{b}-\hat{a} \hat{b}^{\dagger}\right)\right]$ and $\hat{D}^{B}(\alpha)=$ $\exp \left(\alpha \hat{b}^{\dagger}-\alpha^{*} \hat{b}\right)$ are the operators for a beamsplitter and a displacement of mode $B$, where $\left\{\hat{a}, \hat{a}^{\dagger}\right\}$ and $\left\{\hat{b}, \hat{b}^{\dagger}\right\}$ are

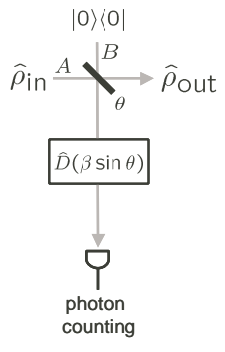

(a)

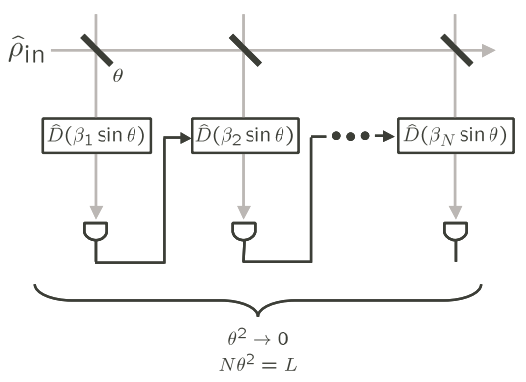

(b)
FIG. 2: Continuous photon counting with displacement operations. (a) Photodetection with a displacement operation. The beamsplitter is parametrized by $\theta$. (b) A sequential photodetection with displacement operations in the limit of small $\theta$ (small reflectance) and a large number of measurement steps.

the annihilation and creation operators for modes $A$ and $B$, respectively. Although photodetection is described by a set of projection operators on the Fock basis $\left\{\hat{\Pi}_{k}=\right.$ $|k\rangle\langle k|\}$, we assume that the parameter $\theta$ is sufficiently small such that the probabilities of detecting more than two photons are negligible. The complex number $\beta$ in the displacement operator will be appropriately determined later. As it is well known, this displacement operation can be realized using a beamsplitter $\hat{B}(\theta)$ and a local oscillator of amplitude $\beta$. For a pure state input $\hat{\rho}_{\text {in }}=$ $\left|\psi_{\text {in }}\right\rangle\left\langle\psi_{\text {in }}\left|, \hat{\rho}_{\text {out }}^{\prime}=\right| \psi_{\text {out }}^{\prime}\right\rangle\left\langle\psi_{\text {out }}^{\prime}\right|$ is given by

$$
\begin{aligned}
\left|\psi_{\text {out }}^{\prime}\right\rangle= & \hat{D}^{B}(\beta \sin \theta) \hat{B}^{A B}(\theta)\left|\psi_{\text {in }}^{A}\right\rangle\left|0^{B}\right\rangle \\
= & e^{-\frac{1}{2}|\beta|^{2} \sin ^{2} \theta} e^{\hat{b}^{\dagger} \beta \sin \theta} e^{-\hat{b} \beta^{*} \sin \theta} e^{-\hat{a} \hat{b}^{\dagger} \tan \theta} \\
& \times e^{\hat{a}^{\dagger} \hat{a} \ln \cos \theta} e^{-\hat{b}^{\dagger} \hat{b} \ln \cos \theta} e^{\hat{a}^{\dagger} \hat{b} \tan \theta}\left|\psi_{\text {in }}^{A}\right\rangle\left|0^{B}\right\rangle,
\end{aligned}
$$

where we applied the Baker-Campbell-Hausdorff (BCH) formula and for $\hat{B}^{A B}(\theta)$ an anti-normally ordered decomposition formula such that $e^{\theta\left(\hat{a}^{\dagger} \hat{b}-\hat{a} \hat{b}^{\dagger}\right)}=$ $e^{-\hat{a}^{\dagger} \tan \theta} e^{\ln \cos \theta\left(\hat{a}^{\dagger} \hat{a}-\hat{b}^{\dagger} \hat{b}\right)} e^{\hat{a}^{\dagger} \hat{b} \tan \theta}$. Throughout, we use the notation $\left|\psi_{\text {in }}^{A}\right\rangle\left|0^{B}\right\rangle \equiv\left|\psi_{\text {in }}^{A}\right\rangle \otimes\left|0^{B}\right\rangle$ etc.

Equation (3) can be further simplified to

$$
\begin{aligned}
\left|\psi_{\text {out }}^{\prime}\right\rangle= & e^{-\frac{1}{2}|\beta|^{2} \sin ^{2} \theta} e^{(\beta \sin \theta-\hat{a} \tan \theta) \hat{b}^{\dagger}} \\
& \times e^{\hat{a} \beta^{*} \frac{\sin ^{2} \theta}{\cos \theta}} e^{\hat{a}^{\dagger} \hat{a} \ln \cos \theta}\left|\psi_{\text {in }}^{A}\right\rangle\left|0^{B}\right\rangle .
\end{aligned}
$$

Here we applied again the $\mathrm{BCH}$ formula such that $e^{-\hat{b} \beta^{*} \sin \theta} e^{-\hat{a} \hat{b}^{\dagger} \tan \theta}=e^{-\hat{a} \hat{b}^{\dagger} \tan \theta} e^{\hat{a} \beta^{*} \frac{\sin ^{2} \theta}{\cos \theta}} e^{-\hat{b} \beta^{*} \sin \theta}$, and we used $\hat{b}^{\dagger} \hat{b}\left|0^{B}\right\rangle=\hat{b}\left|0^{B}\right\rangle=0$.

Let us assume that the photodetector detects at most one photon. We will now consider the two possible outcomes, i.e. $\hat{\rho}_{\text {out }}^{\prime}$ is projected on $\hat{\Pi}_{0}$ or $\hat{\Pi}_{1}$. Since these projectors are rank one operators, the conditional output for each operator can be described by

$$
\hat{\rho}_{\text {out }}^{(k)}=\frac{\hat{M}^{(k)} \hat{\rho}_{\text {in }} \hat{M}^{(k) \dagger}}{\operatorname{Tr}_{\mathrm{A}}\left[\hat{M}^{(k)} \hat{\rho}_{\mathrm{in}} \hat{M}^{(k) \dagger}\right]} \quad(k=0,1),
$$


where $\hat{M}^{(k)}$ is a Kraus operator for the $k$-photon detection event. When no photon is detected at the photodetector, the conditional output is given by

$$
\hat{M}^{(0)}\left|\psi_{\text {in }}\right\rangle=e^{-\frac{1}{2}|\beta|^{2} \sin ^{2} \theta} e^{\hat{a} \beta^{*} \frac{\sin ^{2} \theta}{\cos \theta}} e^{\hat{a}^{\dagger} \hat{a} \ln \cos \theta}\left|\psi_{\text {in }}\right\rangle .
$$

Here we consider $N$ successive operations of Eq. (6) with the displacement $\hat{D}\left(\beta_{n} \sin \theta\right)$ representing the $n$-th operation. For example, for $N=2$, we have

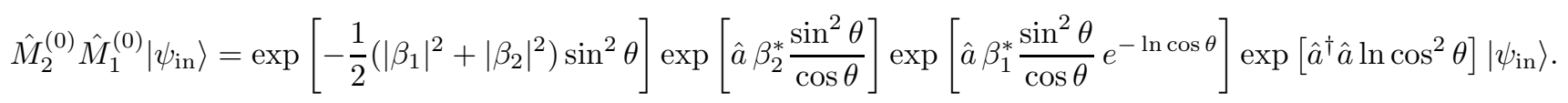

Here we inserted the identity $e^{-\hat{a}^{\dagger} \hat{a} \ln \cos \theta} e^{\hat{a}^{\dagger} \hat{a} \ln \cos \theta}$ and then used $e^{\phi \hat{a}^{\dagger} \hat{a}} \hat{a} e^{-\phi \hat{a}^{\dagger} \hat{a}}=\hat{a} e^{-\phi}$. For $N$ successive operations, the output is then given by

$$
\hat{M}_{N}^{(0)} \hat{M}_{N-1}^{(0)} \cdots \hat{M}_{1}^{(0)}\left|\psi_{\text {in }}\right\rangle=\exp \left[-\frac{1}{2} \sum_{n=1}^{N}\left|\beta_{n}\right|^{2} \sin ^{2} \theta\right] \exp \left[\hat{a} \sum_{n=1}^{N} \beta_{n}^{*} \cos ^{n} \theta \frac{\sin ^{2} \theta}{\cos ^{N+1} \theta}\right] \exp \left[\hat{a}^{\dagger} \hat{a} \ln \cos ^{N} \theta\right]\left|\psi_{\text {in }}\right\rangle .
$$

Let us define the constant parameter $L=N \theta^{2}$ and take the limit $\theta^{2} \rightarrow 0$ and $N \rightarrow \infty$. The displacement operations may now be described in a continuous way via

$$
\begin{aligned}
& \sum_{n=1}^{N}\left|\beta_{n}\right|^{2} \sin ^{2} \theta \approx \sum_{n=1}^{N}\left|\beta_{n}\right|^{2} \frac{L}{N} \rightarrow \int_{0}^{L} \mathrm{~d} l|\beta(l)|^{2} \\
& \sum_{n=1}^{N} \beta_{n}^{*} \cos ^{n} \theta \frac{\sin ^{2} \theta}{\cos \theta} \approx \sum_{n=1}^{N} \beta_{n}^{*}\left(\cos ^{N} \theta\right)^{\frac{n}{N}} \theta^{2}=\sum_{n=1}^{N} \beta_{n}^{*} e^{-\frac{n L}{2 N}} \frac{L}{N} \rightarrow \int_{0}^{L} \mathrm{~d} l \beta^{*}(l) e^{-\frac{l}{2}} .
\end{aligned}
$$

Using these relations, we obtain the output state

$$
\hat{M}_{N}^{(0)} \hat{M}_{N-1}^{(0)} \cdots \hat{M}_{1}^{(0)}\left|\psi_{\text {in }}\right\rangle=\exp \left[-\frac{1}{2} \int_{0}^{L} \mathrm{~d} l|\beta(l)|^{2}\right] \exp \left[\hat{a} e^{\frac{L}{2}} \int_{0}^{L} \mathrm{~d} l \beta^{*}(l) e^{-\frac{l}{2}}\right] \exp \left[-\frac{L}{2} \hat{a}^{\dagger} \hat{a}\right]\left|\psi_{\text {in }}\right\rangle
$$

More generally, when no photon is detected from the $L_{0}$-th detector to the $L_{1}$-th detector, we obtain the output state

$$
\hat{\rho}_{\text {out }}=\frac{\hat{M}_{L_{1}}^{(0)} \hat{M}_{L_{1}-1}^{(0)} \cdots \hat{M}_{L_{0}}^{(0)} \hat{\rho}_{\text {in }} \hat{M}_{L_{0}}^{(0) \dagger} \cdots \hat{M}_{L_{1}-1}^{(0) \dagger} \hat{M}_{L_{1}}^{(0) \dagger}}{\operatorname{Tr}\left[\hat{M}_{L_{1}}^{(0)} \hat{M}_{L_{1}-1}^{(0)} \cdots \hat{M}_{L_{0}}^{(0)} \hat{\rho}_{\text {in }} \hat{M}_{L_{0}}^{(0) \dagger} \cdots \hat{M}_{L_{1}-1}^{(0) \dagger} \hat{M}_{L_{1}}^{(0) \dagger}\right]}=\frac{\hat{S}_{L_{1}-L_{0}} \hat{\rho}_{\text {in }} \hat{S}_{L_{1}-L_{0}}^{\dagger}}{\operatorname{Tr}\left[\hat{S}_{L_{1}-L_{0}} \hat{\rho}_{\mathrm{in}} \hat{S}_{L_{1}-L_{0}}^{\dagger}\right]},
$$

where

$$
\hat{S}_{L_{1}-L_{0}}=\exp \left[\hat{a} e^{\frac{L_{1}-L_{0}}{2}} \int_{L_{0}}^{L_{1}} \mathrm{~d} l \beta^{*}(l) e^{-\frac{l}{2}}\right] \exp \left[-\frac{L_{1}-L_{0}}{2} \hat{a}^{\dagger} \hat{a}\right]
$$

However, when a photon is detected at the $L$-th photodetector, the conditional operation is described by

$$
\hat{M}_{L}^{(1)}\left|\psi_{\text {in }}\right\rangle=\exp \left[-\frac{1}{2}\left|\beta_{L}\right|^{2} \sin ^{2} \theta\right]\left(\beta_{L} \sin \theta-\hat{a} \tan \theta\right) \exp \left[\hat{a} \beta_{L}^{*} \frac{\sin ^{2} \theta}{\cos \theta}\right] \exp \left[\hat{a}^{\dagger} \hat{a} \ln \cos \theta\right]\left|\psi_{\text {in }}\right\rangle
$$

Upon taking the limit $\theta^{2} \rightarrow 0$ and using the continuous representation given in Eq. (9), all the exponential terms in Eq. (13) approach unity. The output now becomes

$$
\hat{\rho}_{\text {out }}=\frac{\hat{M}_{L}^{(1)} \hat{\rho}_{\text {in }} \hat{M}_{L}^{(1) \dagger}}{\operatorname{Tr}\left[\hat{M}_{L}^{(1)} \hat{\rho}_{\text {in }} \hat{M}_{L}^{(1) \dagger}\right]}=\frac{\hat{J}_{L} \hat{\rho}_{\text {in }} \hat{J}_{L}^{\dagger}}{\operatorname{Tr}\left[\hat{J}_{L} \hat{\rho}_{\text {in }} \hat{J}_{L}^{\dagger}\right]}
$$

where $\hat{M}_{L}^{(1)}=\theta \hat{J}_{L}$ and

$$
\hat{J}_{L}=\beta(L)-\hat{a} .
$$

Using these expressions, a continuous measurement based on beamsplitting, displacement operations, and 
photon counting is described via the operator

$$
\hat{S}_{L_{n}-L_{n-1}} \hat{J}_{L_{n-1}} \hat{S}_{L_{n-1}-L_{n-2}} \cdots \hat{J}_{L_{1}} \hat{S}_{L_{1}-0} .
$$

This expression can also be obtained as a solution of the master equation of the system and this kind of conditional dynamics is called quantum jump process in terms of quantum statistics theory [28]. Although analytical solutions can be obtained in our case, it should be noted when the system has complicated quantum jump e.g. the measurement has continuous outcome, one effective approach is the stochastic unraveling of the master equation. One of the successful applications of this approach is shown in Ref. [29].

In the next section, we will show how a given projective measurement can be implemented via the apparatus discussed here. Finally, we note that when $L$ is replaced by a time parameter $T$, our formulation is equivalent to the conventional time domain continuous measurement model including displacement operations [18].

\section{PROJECTIVE MEASUREMENTS VIA CONTINUOUS MEASUREMENT}

Projective measurements represent an important special case among the generalized measurements. In this section, we show that the Dolinar receiver can be systematically derived from an orthogonality condition, similar to that used for analyzing the exact distinguishability of orthogonal states in a projection measurement [20]. First, we will apply this approach to the original Dolinar receiver, that is, the discrimination of binary coherent states. In a further example, we examine a binary projection measurement onto a photonic-qubit basis, which otherwise cannot be implemented with linear optics including photon counting, finite steps of conditional dynamics, and arbitrary auxiliary photon states.

Before discussing particular examples, we briefly summarize our approach. The problem of implementing a complete projection measurement $\left\{\Pi_{i}=\left|\pi_{i}\right\rangle\left\langle\pi_{i}\right|\right\}$ can be regarded as the problem of an exact discrimination of the orthogonal signal states $\left|\pi_{i}\right\rangle[20$ ]. In order to achieve an exact discrimination, these signal states, when conditionally transformed via partial measurements and feedforward, must remain orthogonal after each step of the intermediate measurements. In our approach, these measurements are assumed to be arbitrarily weak, asymptotically corresponding to a continuous measurement. Via the orthogonality constraint, we can infer the input signal by counting the total number of detected photons $N_{\text {tot }}$. In the limit of infinitely many photodetection steps, the final state must be in a vacuum state $|0\rangle$ due to the energy loss at each step [30]. Combining this fact with the condition that the signals always remain mutually orthogonal, we know that every possible result of the $N_{\text {tot}}$-photon detection can be triggered only by one of the two signal states with nonzero probability. Eventually, we can infer the input signal state perfectly by counting $N_{\text {tot }}$.

\section{A. Minimum error discrimination of binary coherent states}

The minimum error detection of the binary coherent states $\{|\alpha\rangle,|-\alpha\rangle\}$ is achieved via the projection operators corresponding to the orthogonal states

$$
\begin{aligned}
& \left|\omega_{0}\right\rangle=\sqrt{\frac{1-P_{e}}{1-\kappa^{2}}}|\alpha\rangle-\sqrt{\frac{P_{e}}{1-\kappa^{2}}}|-\alpha\rangle, \\
& \left|\omega_{1}\right\rangle=\sqrt{\frac{P_{e}}{1-\kappa^{2}}}|\alpha\rangle-\sqrt{\frac{1-P_{e}}{1-\kappa^{2}}}|-\alpha\rangle,
\end{aligned}
$$

where $\kappa=|\langle\alpha \mid-\alpha\rangle|$ and

$$
P_{e}=\frac{1}{2}\left(1-\sqrt{1-\kappa^{2}}\right),
$$

is the minimum error probability. For the sake of simplicity, we assume that the a priori probabilities for the signals are equal.

Let us first prove that the projection onto $\left\{\left|\omega_{0}\right\rangle,\left|\omega_{1}\right\rangle\right\}$ cannot be implemented using linear optics, finite steps of conditional dynamics, and arbitrary auxiliary states. As for the detection mechanisms, we may restrict ourselves to photon counting since homodyne detection with linear optics never leads to non-Gaussian operation, as required for our projection measurement. Now, using the criteria for exact discrimination of orthogonal states [20], one finds that already the detection of a first output mode, after mixing the signal mode with the auxiliary modes, inevitably destroys the orthogonality. Defining an arbitrary auxiliary state $|A\rangle$ with arbitrarily many modes, the necessary conditions for preserving the orthogonality after such a first detection (and hence for potentially enabling one to exactly discriminate the states via further detections and conditional transformations) are 20.

$$
\left\langle A\left|\left\langle\omega_{0}\left|\left(\hat{c}^{\dagger}\right)^{n} \hat{c}^{n}\right| \omega_{1}\right\rangle\right| A\right\rangle=0, \quad \forall n=0,1,2, \ldots
$$

Here, the 0th order $(n=0)$ just corresponds to the orthogonality of the signal states. The annihilation operator $\hat{c}$ represents the first mode being detected after the linear-optics transformation. This output mode may be decomposed into two parts of which one refers to the signal mode and the other one to the auxiliary modes,

$$
\hat{c}=\nu_{1} \hat{a}_{1}+b_{\text {aux }} \hat{c}_{\text {aux }}+\gamma .
$$

Here, $\nu_{1} \equiv U_{j 1}$ is the complex entry of the unitary matrix $U$ for describing the linear-optics mixing of the mode $j$ to be detected with the signal mode 1 . The mixing of mode $j$ with the auxiliary modes due to linear optics is described by the annihilation operator $\hat{c}_{\text {aux }}$, where $b_{\text {aux }}$ is a real parameter. The complex parameter $\gamma$ enables us to include the possibility of phase-space displacements before the detection 31. Note that without including displacements and for signal states with a fixed number of photons, arbitrary auxiliary states $|A\rangle$ cannot help to 
provide nontrivial solutions to the conditions in Eq. (20), if there are only trivial solutions without an extra state $|A\rangle$ [20]. For the projection onto $\left\{\left|\omega_{0}\right\rangle,\left|\omega_{1}\right\rangle\right\}$, however, the signal states have an unfixed photon number. Thus, using an auxiliary state $|A\rangle$ having unfixed number too [20], or employing a nonzero phase-space displacement $\gamma \neq 0$ may indeed help.

After some algebra, for the first-order condition $n=1$ from Eq. (20) using Eq. (21), we obtain

$$
\left|\nu_{1}\right|^{2}|\alpha|^{2}=i \sqrt{1-\kappa^{2}} \operatorname{Im} \delta
$$

where $\alpha \neq 0$ is the complex amplitude given by the signal states, the parameter $\kappa=|\langle\alpha \mid-\alpha\rangle|$ is also defined through the signal states, and $\operatorname{Im} \delta$ is the imaginary part of

$$
\delta \equiv \nu_{1}^{*} \alpha^{*}\left(\gamma+\left\langle A\left|b_{\mathrm{aux}} \hat{c}_{\mathrm{aux}}\right| A\right\rangle\right)
$$

Since $\kappa^{2}<1$ for $\alpha \neq 0$, one can easily see that the only solution to the condition in Eq. (22) is trivial, $\nu_{1}=0$. In other words, only if there is no mixing at all between the mode $j$ to be detected and the signal mode 1 , the orthogonality is (trivially) preserved. For any finite mixing between the modes $j$ and 1, exact discrimination of the signal states is no longer possible. In our approach based upon continuous measurement, every single conditionaldynamics step is supposed to be arbitrarily weak corresponding to the limit $\nu_{1} \rightarrow 0$. Thus, in this limit, the orthogonality condition may be satisfied. However, typically, such a scheme does not provide any information about the input signal states. Yet in the following, we demonstrate that by combining infinitely many arbitrarily weak detections of the signal mode, corresponding to a continuous measurement of the signal mode, eventually perfect state discrimination can be accomplished.

Suppose that the states $\left\{\left|\omega_{0}\right\rangle,\left|\omega_{1}\right\rangle\right\}$ are sent into the continuous measurement apparatus discussed in the preceding section. When no photon is counted during $\left[0, L_{1}\right)$, the signals evolve as

$$
\begin{aligned}
\hat{S}_{L_{1}-0}\left|\omega_{0}\right\rangle= & \sqrt{\frac{1-P_{e}}{1-\kappa^{2}}} \exp [B]\left|\alpha e^{-\frac{L_{1}}{2}}\right\rangle \\
& -\sqrt{\frac{P_{e}}{1-\kappa^{2}}} \exp [-B]\left|-\alpha e^{-\frac{L_{1}}{2}}\right\rangle, \\
\hat{S}_{L_{1}-0}\left|\omega_{1}\right\rangle= & \sqrt{\frac{P_{e}}{1-\kappa^{2}}} \exp [B]\left|\alpha e^{-\frac{L_{1}}{2}}\right\rangle \\
& -\sqrt{\frac{1-P_{e}}{1-\kappa^{2}}} \exp [-B] \mid-\alpha e^{\left.-\frac{L_{1}}{2}\right\rangle}
\end{aligned}
$$

where

$$
B=\alpha \int_{0}^{L_{1}} \mathrm{~d} l \beta^{*}(l) e^{-\frac{l}{2}}
$$

We note that these states are unnormalized. By calculating the inner product between Eqs. (24) and (25), we can find the condition that these signals are orthogonal,

$$
\begin{aligned}
& \sqrt{P_{e}\left(1-P_{e}\right)}\left\{\exp \left[B+B^{*}\right]+\exp \left[-\left(B+B^{*}\right)\right]\right\} \\
& -\left\{P_{e} \exp \left[B-B^{*}\right]+\left(1-P_{e}\right) \exp \left[-\left(B-B^{*}\right)\right]\right\} \\
& \times \exp \left[-2|\alpha|^{2} e^{-L_{1}}\right]=0
\end{aligned}
$$

From the orthogonality condition Eq. (27) with Eq. (26), we obtain the function for the displacement operation

$$
\beta(l)= \pm \frac{\alpha e^{-\frac{l}{2}}}{\sqrt{1-\exp \left[-4|\alpha|^{2}\left(1-e^{-l}\right)\right]}} .
$$

By defining the two solutions in Eq. (28) as $\beta_{+}(l), \beta_{-}(l)$, respectively, we can derive the relations

$\exp \left[\alpha \int_{L_{j}}^{L_{j+1}} \mathrm{~d} l \beta_{+}(l) e^{-\frac{l}{2}}\right]=\frac{e^{-|\alpha|^{2} e^{-L_{j+1}}}}{e^{-|\alpha|^{2} e^{-L_{j}}}} \frac{\sqrt{1-P_{e}^{L_{j+1}}}}{\sqrt{1-P_{e}^{L_{j}}}}$,

$$
\exp \left[\alpha \int_{L_{j}}^{L_{j+1}} \mathrm{~d} l \beta_{-}(l) e^{-\frac{l}{2}}\right]=\frac{e^{-|\alpha|^{2} e^{-L_{j+1}}}}{e^{-|\alpha|^{2} e^{-L_{j}}}} \frac{\sqrt{P_{e}^{L_{j+1}}}}{\sqrt{P_{e}^{L_{j}}}}
$$

where

$$
P_{e}^{L}=\frac{1}{2}\left(1-\sqrt{1-\exp \left[-4|\alpha|^{2}\left(1-e^{-L}\right)\right]}\right) .
$$

In the following, we choose $\beta_{+}(l)$ as a displacement function for $\left[0, L_{1}\right)\left(\beta_{-}(l)\right.$ leads to the same conclusions) and assume that a photon is detected at the $L_{1}$-th detector. The equations (24), (25), (29), and (30) yield

$$
\begin{aligned}
\hat{S}_{L_{1}-0}\left|\omega_{0}\right\rangle \propto & \sqrt{1-P_{e}} \frac{\sqrt{1-P_{e}^{L_{1}}}}{\sqrt{1-P_{e}^{0}}}\left|\alpha e^{-\frac{L_{1}}{2}}\right\rangle \\
& -\sqrt{P_{e}} \frac{\sqrt{P_{e}^{L_{1}}}}{\sqrt{P_{e}^{0}}}\left|-\alpha e^{-\frac{L_{1}}{2}}\right\rangle, \\
\hat{S}_{L_{1}-0}\left|\omega_{1}\right\rangle \propto & \sqrt{P_{e}} \frac{\sqrt{1-P_{e}^{L_{1}}}}{\sqrt{1-P_{e}^{0}}}\left|\alpha e^{-\frac{L_{1}}{2}}\right\rangle \\
& -\sqrt{1-P_{e}} \frac{\sqrt{P_{e}^{L_{1}}}}{\sqrt{P_{e}^{0}}}\left|-\alpha e^{-\frac{L_{1}}{2}}\right\rangle,
\end{aligned}
$$

where unimportant global coefficients are omitted. As for the states after detecting a photon, by using the relations

$$
\beta_{+}\left(L_{1}\right)-\alpha e^{-\frac{L_{1}}{2}}=\frac{2 P_{e}^{L_{1}} \alpha e^{-\frac{L_{1}}{2}}}{\sqrt{1-\exp \left[-4|\alpha|^{2}\left(1-e^{-L_{1}}\right)\right]}}
$$

$$
\beta_{+}\left(L_{1}\right)+\alpha e^{-\frac{L_{1}}{2}}=\frac{2\left(1-P_{e}^{L_{1}}\right) \alpha e^{-\frac{L_{1}}{2}}}{\sqrt{1-\exp \left[-4|\alpha|^{2}\left(1-e^{-L_{1}}\right)\right]}}
$$


we find

$$
\begin{aligned}
\hat{J}_{L_{1}} \hat{S}_{L_{1}-0}\left|\omega_{0}\right\rangle \propto & \sqrt{1-P_{e}} \frac{\sqrt{1-P_{e}^{L_{1}}}}{\sqrt{1-P_{e}^{0}}} P_{e}^{L_{1}}\left|\alpha e^{-\frac{L_{1}}{2}}\right\rangle \\
& -\sqrt{P_{e}} \frac{\sqrt{P_{e}^{L_{1}}}}{\sqrt{P_{e}^{0}}}\left(1-P_{e}^{L_{1}}\right)\left|-\alpha e^{-\frac{L_{1}}{2}}\right\rangle \\
\propto & \sqrt{1-P_{e}} \frac{\sqrt{P_{e}^{L_{1}}}}{\sqrt{1-P_{e}^{0}}}\left|\alpha e^{-\frac{L_{1}}{2}}\right\rangle \\
& -\sqrt{P_{e}} \frac{\sqrt{1-P_{e}^{L_{1}}}}{\sqrt{P_{e}^{0}}}\left|-\alpha e^{-\frac{L_{1}}{2}}\right\rangle,
\end{aligned}
$$

and similarly,

$$
\begin{aligned}
\hat{J}_{L_{1}} \hat{S}_{L_{1}-0}\left|\omega_{1}\right\rangle \propto & \sqrt{P_{e}} \frac{\sqrt{P_{e}^{L_{1}}}}{\sqrt{1-P_{e}^{0}}}\left|\alpha e^{-\frac{L_{1}}{2}}\right\rangle \\
& -\sqrt{1-P_{e}} \frac{\sqrt{1-P_{e}^{L_{1}}}}{\sqrt{P_{e}^{0}}}\left|-\alpha e^{-\frac{L_{1}}{2}}\right\rangle .
\end{aligned}
$$

Comparing them with Eqs. (32) and (33), we can easily see that these states are still orthogonal to each other. Also, we can derive the relations,

$$
\begin{aligned}
& \hat{J}_{L_{j+1}} \hat{S}_{L_{j+1}-L_{j}}\left|\alpha e^{-\frac{L_{j}}{2}}\right\rangle \\
& =\frac{1}{2} e^{-4|\alpha|^{2}\left(1-e^{-L_{j+1}}\right)} \frac{\sqrt{P_{e}^{L_{j+1}}}}{\sqrt{1-P_{e}^{L_{j}}}}\left|\alpha e^{-\frac{L_{j+1}}{2}}\right\rangle, \\
& \hat{J}_{L_{j+1}} \hat{S}_{L_{j+1}-L_{j}\left|-\alpha e^{-\frac{L_{j}}{2}}\right\rangle} \\
& =\frac{1}{2} e^{-4|\alpha|^{2}\left(1-e^{-L_{j+1}}\right)} \frac{\sqrt{1-P_{e}^{L_{j+1}}}}{\sqrt{P_{e}^{L_{j}}}}\left|-\alpha e^{-\frac{L_{j+1}}{2}}\right\rangle,
\end{aligned}
$$

for the measurement process with the displacement function $\beta_{+}(l)$, and

$$
\begin{aligned}
& \hat{J}_{L_{j+1}} \hat{S}_{L_{j+1}-L_{j}}\left|\alpha e^{-\frac{L_{j}}{2}}\right\rangle \\
& =\frac{1}{2} e^{-4|\alpha|^{2}\left(1-e^{-L_{j+1}}\right)} \frac{\sqrt{1-P_{e}^{L_{j+1}}}}{\sqrt{P_{e}^{L_{j}}}}\left|\alpha e^{-\frac{L_{j+1}}{2}}\right\rangle, \\
& \hat{J}_{L_{j+1}} \hat{S}_{L_{j+1}-L_{j}\left|-\alpha e^{-L_{j}}\right\rangle} \\
& =\frac{1}{2} e^{-4|\alpha|^{2}\left(1-e^{-L_{j+1}}\right)} \frac{\sqrt{P_{e}^{L_{j+1}}}}{\sqrt{1-P_{e}^{L_{j}}}}\left|-\alpha e^{-\frac{L_{j+1}}{2}}\right\rangle,
\end{aligned}
$$

for the measurement process with the displacement function $\beta_{-}(l)$, where $\left(P_{e}^{L_{j+1}}\left(1-P_{e}^{L_{j+1}}\right)\right)^{1 / 2}=$ $e^{-4|\alpha|^{2}\left(1-e^{-L_{j+1}}\right) / 2}$

From Eqs. (29), (30), (36) and (37), we can determine the local oscillator function $\beta(l)$ for the next zero photon counting process $\hat{S}_{L_{2}-L_{1}}$. In our case, the orthogonality condition requires using $\beta_{-}(l)$ for $\hat{S}_{L_{2}-L_{1}}$, as the output states $\hat{S}_{L_{2}-L_{1}} \hat{J}_{L_{1}} \hat{S}_{L_{1}-0}\left|\omega_{i}\right\rangle(i=0,1)$ have the similar structure as in Eqs. (36) and (37), respectively, except that $L_{1}$ is replaced by $L_{2}$. Eventually, one can preserve the orthogonality by switching the sign of the displacement function $\beta_{ \pm}(l)$ each time a photon is detected. After applying many of these operations, the signals

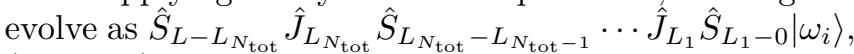
$(i=0,1)$, where $N_{\text {tot }}$ is the total number of detected photons $\left(N_{\text {tot }}=0,1 \cdots, \infty\right)[32$ and we will take an infinitely large $L$. The final states are now derived with the help of Eqs. (38), (39), (40), and (41), and classified via the parity of $N_{\text {tot }}$. When $N_{\text {tot }}$ is zero or even, the output state for the input $\left|\omega_{0}\right\rangle$ is given by

$$
\begin{aligned}
& \left|\omega_{0}^{L}\right\rangle=\hat{S}_{L-L_{N_{\text {tot }}}} \hat{J}_{L_{N_{\text {tot }}}} \hat{S}_{L_{N_{\text {tot }}}-L_{N_{\text {tot }}-1}} \cdots \hat{J}_{L_{1}} \hat{S}_{L_{1}-0}\left|\omega_{0}\right\rangle
\end{aligned}
$$

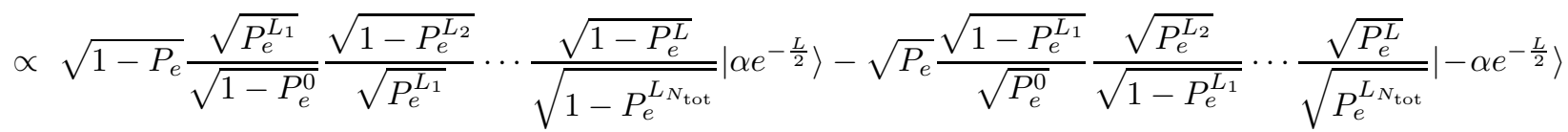

$$
\begin{aligned}
& \propto \sqrt{1-P_{e}} \sqrt{1-P_{e}^{L}}\left|\alpha e^{-\frac{L}{2}}\right\rangle-\sqrt{P_{e}} \sqrt{P_{e}^{L}}\left|-\alpha e^{-\frac{L}{2}}\right\rangle \\
& =e^{-\frac{1}{2}|\alpha|^{2} e^{-L}} \sum_{m=0}^{\infty} \frac{1}{\sqrt{m !}} \begin{cases}\left(\sqrt{1-P_{e}} \sqrt{1-P_{e}^{L}}-\sqrt{P_{e}} \sqrt{P_{e}^{L}}\right)\left(\alpha e^{-\frac{L}{2}}\right)^{m}|m\rangle & m \text { is zero or even } \\
\left(\sqrt{1-P_{e}} \sqrt{1-P_{e}^{L}}+\sqrt{P_{e}} \sqrt{P_{e}^{L}}\right)\left(\alpha e^{-\frac{L}{2}}\right)^{m}|m\rangle & m \text { is odd }\end{cases} \\
& =\left(\sqrt{1-e^{-4|\alpha|^{2}}}+\frac{|\alpha|^{2} e^{-4|\alpha|^{2}}}{\sqrt{1-e^{-4|\alpha|^{2}}}} e^{-L} \cdots\right)|0\rangle+(1+\cdots) \alpha e^{-\frac{L}{2}}|1\rangle+\frac{1}{\sqrt{2}}\left(\sqrt{1-e^{-4|\alpha|^{2}}}+\cdots\right) \alpha^{2} e^{-L}|2\rangle+\cdots,
\end{aligned}
$$

where $|m\rangle$ is the $m$-photon number state and note that $P_{e}^{0}=1 / 2$ and $\lim _{L \rightarrow \infty} P_{e}^{L}=P_{e}$. In the last line, the terms 
only up to the order of $e^{-L}$ are given. Similarly, for the input $\left|\omega_{1}\right\rangle$, we obtain

$$
\begin{aligned}
\left|\omega_{1}^{L}\right\rangle & =\hat{S}_{L-L_{N_{\mathrm{tot}}}} \hat{J}_{L_{\mathrm{t}_{\mathrm{tot}}}} \hat{S}_{L_{N_{\mathrm{tot}}}-L_{N_{\mathrm{tot}}-1} \cdots \hat{J}_{L_{1}} \hat{S}_{L_{1}-0}\left|\omega_{1}\right\rangle} \\
& \propto \sqrt{P_{e}} \sqrt{1-P_{e}^{L}}\left|\alpha e^{-\frac{L}{2}}\right\rangle-\sqrt{1-P_{e}} \sqrt{P_{e}^{L}}\left|-\alpha e^{-\frac{L}{2}}\right\rangle \\
& =e^{-\frac{1}{2}|\alpha|^{2} e^{-L}} \sum_{m=0}^{\infty} \frac{1}{\sqrt{m !}}\left\{\begin{array}{c}
\left(\sqrt{P_{e}} \sqrt{1-P_{e}^{L}}-\sqrt{1-P_{e}} \sqrt{P_{e}^{L}}\right)\left(\alpha e^{-\frac{L}{2}}\right)^{m}|m\rangle \quad m \text { is zero or even } \\
\left(\sqrt{P_{e}} \sqrt{1-P_{e}^{L}}+\sqrt{1-P_{e}} \sqrt{P_{e}^{L}}\right)\left(\alpha e^{-\frac{L}{2}}\right)^{m}|m\rangle \\
\end{array}\right. \\
& =\left(-\frac{|\alpha|^{2} e^{-2|\alpha|^{2}}}{\sqrt{1-e^{-4|\alpha|^{2}}}} e^{-L}+\cdots\right)|0\rangle+\left(e^{-2|\alpha|^{2}}+\cdots\right) \alpha e^{-\frac{L}{2}}|1\rangle+\cdots,
\end{aligned}
$$

where the last line also shows the terms up to the order of $e^{-L}$. From Eqs. (42) and (43), we can check that these two possible outputs are still orthogonal to each other and, even in the approximated form, the orthogonality is satisfied in any order of $L$. For example, the inner product between $\left|\omega_{0}^{L}\right\rangle$ and $\left|\omega_{1}^{L}\right\rangle$ up to the order of $e^{-L}$ is computed as

$$
\begin{aligned}
\left\langle\omega_{0}^{L} \mid \omega_{1}^{L}\right\rangle \approx & -\sqrt{1-e^{-4|\alpha|^{2}}} \frac{|\alpha|^{2} e^{-2|\alpha|^{2}}}{\sqrt{1-e^{-4|\alpha|^{2}}}} e^{-L}\langle 0 \mid 0\rangle \\
& +|\alpha|^{2} e^{-2|\alpha|^{2}} e^{-L}\langle 1 \mid 1\rangle \\
= & 0 .
\end{aligned}
$$

Then, upon taking the limit of $L \rightarrow \infty$ and normalizing Eq. (42), we know that $\left|\omega_{0}^{L}\right\rangle$ approaches the vacuum state. We also find from Eq. (43) that the state $\left|\omega_{1}^{L}\right\rangle$ converges to $|1\rangle$. However, since all the terms in Eq. (43) decrease exponentially for large $L$, we know that $\left|\omega_{1}^{L}\right\rangle$ does not occur at all in the limit of $L \rightarrow \infty$. Hence we have

$$
\begin{aligned}
& \left|\omega_{0}^{L}\right\rangle \rightarrow|0\rangle, \\
& \left|\omega_{1}^{L}\right\rangle \rightarrow 0,
\end{aligned}
$$

and thus, if the number of the totally detected photons $N_{\text {tot }}$ is zero or even, we can unambiguously identify the input state $\left|\omega_{0}\right\rangle$.

On the other hand, when $N_{\text {tot }}$ is odd, the final states are given by

$$
\begin{aligned}
\left|\omega_{0}^{L}\right\rangle & \rightarrow 0, \\
\left|\omega_{1}^{L}\right\rangle & \rightarrow|0\rangle,
\end{aligned}
$$

and hence, in this case, we can unambiguously infer the input state $\left|\omega_{1}\right\rangle$. Let us finally emphasize that our model may be also translated into the time domain by replacing the spatial parameter $l$ by the time $t$. Therefore, our approach provides an alternative derivation of the original Dolinar receiver. This approach is relatively simple, because it only relies upon the preservation of the orthogonality of the signals during the entire measurement process.

\section{B. Photonic-qubit signals}

In this subsection, we show that our measurement model is not only applicable to coherent-state signals, but it may also be applied to other types of signals, for example, photon-number qubits. As an example, let us consider the projection measurement onto the basis $\left|\omega_{0,1}\right\rangle=(|0\rangle \pm|1\rangle) / \sqrt{2}$, for which one can derive a no-go statement in terms of the criteria of Ref. 20].

As for the exact discrimination of $\left|\omega_{0,1}\right\rangle$, let us first briefly discuss the no-go statement for any linear-optics scheme based on photon counting, finite steps of conditional dynamics, and arbitrary auxiliary states. Since the signal states have an unfixed number of photons, using an auxiliary state $|A\rangle$ with unfixed photon number or employing phase-space displacements may help to exactly discriminate the signal states 20 . For the signal states $\left|\omega_{0,1}\right\rangle$, the necessary conditions in any conditionaldynamics scheme become [20]

$$
\left\langle A\left|\left\langle\omega_{0}\left|\left(\hat{c}^{\dagger}\right)^{n} \hat{c}^{n}\right| \omega_{1}\right\rangle\right| A\right\rangle=0, \quad \forall n=0,1,2, \ldots
$$

Here, we use the same definitions as in the preceding section. By inserting the decomposition of Eq. (21) into the first-order $n=1$ condition, we obtain now

$$
\left|\nu_{1}\right|^{2}=2 i \operatorname{Im} \delta
$$

where now $\operatorname{Im} \delta$ is the imaginary part of

$$
\delta \equiv \nu_{1}\left(\gamma^{*}+\left\langle A\left|b_{\text {aux }} \hat{c}_{\text {aux }}^{\dagger}\right| A\right\rangle\right)
$$

Again, only the trivial solution $\nu_{1}=0$ exists. Thus, there is no linear-optics scheme based on a finite first detection step that achieves the exact discrimination of $\left|\omega_{0,1}\right\rangle$. Let us now see how a continuous-measurement based scheme leads to the perfect discrimination of the signal states.

The design for the measurement apparatus is the same as that of the preceding subsection. So we send the states $\left\{\left|\omega_{0}\right\rangle,\left|\omega_{1}\right\rangle\right\}$ into the measurement apparatus. Let us assume that the first photon is detected at the $L_{1}$-th detector and no photon is counted during $\left[0, L_{1}\right)$. The out- 
coming signals are then given by

$$
\hat{S}_{L_{1}-0}\left|\omega_{1}\right\rangle=\left(1-\sqrt{1-e^{-L_{1}}}\right)|0\rangle-e^{-\frac{L_{1}}{2}}|1\rangle .
$$

$$
\begin{aligned}
& \hat{S}_{L_{1}-0}\left|\omega_{0}\right\rangle=\left(1+\int_{0}^{L_{1}} \mathrm{~d} l \beta^{*}(l) e^{-\frac{l}{2}}\right)|0\rangle+e^{-\frac{L_{1}}{2}}|1\rangle, \\
& \hat{S}_{L_{1}-0}\left|\omega_{1}\right\rangle=\left(1-\int_{0}^{L_{1}} \mathrm{~d} l \beta^{*}(l) e^{-\frac{l}{2}}\right)|0\rangle-e^{-\frac{L_{1}}{2}}|1\rangle .
\end{aligned}
$$

The orthogonality condition for Eqs. (52) and (53) implies an appropriate local oscillator function for $\left\{\left|\omega_{0}\right\rangle,\left|\omega_{1}\right\rangle\right\}$, namely

$$
\beta_{L_{1}-0}(l)= \pm \frac{e^{-\frac{l}{2}}}{2 \sqrt{1-e^{-l}}} .
$$

When choosing the plus solution for $\beta_{L_{1}-0}(l)$, Eqs. (52) and (53) can be rewritten as

$$
\hat{S}_{L_{1}-0}\left|\omega_{0}\right\rangle=\left(1+\sqrt{1-e^{-L_{1}}}\right)|0\rangle+e^{-\frac{L_{1}}{2}}|1\rangle,
$$

After detecting a photon at $L_{1}$, the states become

$$
\begin{aligned}
& \hat{J}_{L_{1}} \hat{S}_{L_{1}-0}\left|\omega_{0}\right\rangle \propto\left(1-\sqrt{1-e^{-L_{1}}}\right)|0\rangle+e^{-\frac{L_{1}}{2}}|1\rangle, \\
& \hat{J}_{L_{1}} \hat{S}_{L_{1}-0}\left|\omega_{1}\right\rangle \propto\left(1+\sqrt{1-e^{-L_{1}}}\right)|0\rangle-e^{-\frac{L_{1}}{2}}|1\rangle,
\end{aligned}
$$

and these are still orthogonal to each other.

Let us consider the next interval $\left(L_{1}, L_{2}\right)$, where we assume that the second photon is detected at $L_{2}$. Defining the local oscillator function $\beta_{L_{2}-L_{1}}(l)$, we obtain

$$
\begin{aligned}
& \hat{S}_{L_{2}-L_{1}} \hat{J}_{L_{1}} \hat{S}_{L_{1}-0}\left|\omega_{0}\right\rangle \propto\left(1-\sqrt{1-e^{-L_{1}}}+e^{-\frac{L_{1}}{2}} \int_{L_{1}}^{L_{2}} \mathrm{~d} l \beta_{L_{2}-L_{1}}^{*}(l) e^{-\frac{l}{2}}\right)|0\rangle+e^{-\frac{L_{2}}{2}}|1\rangle, \\
& \hat{S}_{L_{2}-L_{1}} \hat{J}_{L_{1}} \hat{S}_{L_{1}-0}\left|\omega_{1}\right\rangle \propto\left(1+\sqrt{1-e^{-L_{1}}}-e^{-\frac{L_{1}}{2}} \int_{L_{1}}^{L_{2}} \mathrm{~d} l \beta_{L_{2}-L_{1}}^{*}(l) e^{-\frac{l}{2}}\right)|0\rangle-e^{-\frac{L_{2}}{2}}|1\rangle .
\end{aligned}
$$

Now the orthogonality condition implies

$$
\beta_{L_{2}-L_{1}}(l)=-\frac{e^{-\frac{l-L_{1}}{2}}}{2 \sqrt{1-e^{-l}}}
$$

More generally, the local oscillator function for the interval $\left(L_{j}, L_{j+1}\right)$ is given by

$$
\beta_{L_{j+1}-L_{j}}(l)=(-1)^{j} \frac{e^{-\frac{l-L_{j}}{2}}}{2 \sqrt{1-e^{-l}}}, \quad\left(L_{0}=0\right) .
$$

Using Eq. (62), the signal states after the whole detection process are described by

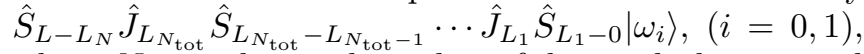
where $N_{\text {tot }}$ is the total number of detected photons and $\left(L \rightarrow \infty, N_{\text {tot }}=0,1 \cdots, \infty\right)$. When $N_{\text {tot }}$ is zero or even, the final states are

$$
\begin{aligned}
\left|\omega_{0}^{L}\right\rangle & \propto\left(1+\sqrt{1-e^{-L}}\right)|0\rangle+e^{-\frac{L}{2}}|1\rangle \\
& =\left(2-\frac{1}{2} e^{-L}-\cdots\right)|0\rangle+e^{-\frac{L}{2}}|1\rangle \\
& \rightarrow|0\rangle \\
\left|\omega_{1}^{L}\right\rangle & \propto\left(1-\sqrt{1-e^{-L}}\right)|0\rangle-e^{-\frac{L}{2}}|1\rangle \\
& =\left(\frac{1}{2} e^{-L}+\cdots\right)|0\rangle-e^{-\frac{L}{2}}|1\rangle \\
& \rightarrow 0 .
\end{aligned}
$$

These are still orthogonal to each other in any order of $L$. On the other hand, when $N_{\text {tot }}$ is odd, we have

$$
\begin{aligned}
& \left|\omega_{0}^{L}\right\rangle \rightarrow 0, \\
& \left|\omega_{1}^{L}\right\rangle \rightarrow|0\rangle .
\end{aligned}
$$

Eventually, one finds that it is possible to distinguish $\left|\omega_{0}\right\rangle$ and $\left|\omega_{1}\right\rangle$ perfectly by checking the parity of the totally detected photon number. Let us finally mention that 
the projection onto arbitrary orthogonal superpositions of the vacuum state $|0\rangle$ and the single photon state $|1\rangle$,

$$
\begin{aligned}
& \left|\omega_{0}\right\rangle=f_{0}|0\rangle+f_{1} e^{i \varphi}|1\rangle, \\
& \left|\omega_{1}\right\rangle=f_{1}|0\rangle-f_{0} e^{i \varphi}|1\rangle,
\end{aligned}
$$

can be achieved in the same manner as described above.

\section{CONCLUSIONS}

In summary, we systematically derived a scheme which is equivalent to the original Dolinar receiver for implementing the minimum error discrimination of binary coherent-state signals. This scheme corresponds to a spatial version of the Dolinar receiver, based upon linear optics, photodetectors, continuous measurement and feedforward. In our approach, as opposed to previous works, we focus on the (asymptotically) perfect implementation of a given projection measurement. In order to derive the Dolinar-type scheme, we consider the projection measurement that corresponds to the minimum error discrimination of binary coherent-state signals. The derivation then relies on the constraint that, for discriminating the orthogonal states of the measurement basis, the conditional states in each detection and feedforward step must remain orthogonal. This new derivation method does not require complicated optimization procedures and is applicable to various kinds of projection measurements. We showed that not only the particular measurement associated with the coherent-state signals treated in Dolinar's original proposal, but also other types of projection measurements can be implemented via continuous measurement and feedforward. Significantly, in our approach, optimal measurement performance, i.e., the maximum measurement efficiency allowed by quantum mechanics, is achieved without the need of expensive entangled auxiliary resources. However, finite feedforward plus arbitrarily many auxiliary photons must be replaced by arbitrarily fast feedforward based on arbitrarily weak measurements.

Although we considered only projection measurements of a single-mode field in this paper, our methodology might be applicable to more general scenarios as well, including generalized measurements or joint projective measurements for more modes. While the possibility of a linear-optics implementation of such scenarios has been studied already [20, 33], it remains an open question whether the approach based on continuous measurement and feedforward allows for an optimal efficiency also in these more general schemes.

Finally, an important question is whether our scheme can be used in a real, necessarily finite, physical implementation. In order to address this question, one would have to consider a discrete analogue of our scheme, including finite feedforward and weak, but finite measurements. One may then determine bounds in terms of suitable figures of merit, e.g. the minimum average error or the maximum success probability, for given feedforward resources.

\section{Acknowledgments}

The authors thank M. Ban for helpful discussions. This work was supported by the DFG under the EmmyNoether program, the EU FET network RAMBOQ and the network of competence QIP of the state of Bavaria.
[1] C. H. Bennett, G. Brassard, C. Crépeau, R. Jozsa, A. Peres, and W. K. Wootters Phys. Rev. Lett. 70, 1895 (1993).

[2] N. Gisin, G. Ribordy, W. Tittel, and H. Zbinden, Rev. Mod. Phys. 74, 145 (2002).

[3] P. Hausladen, R. Jozsa, B. Schumacher, M. Westmoreland, and W. K. Wootters, Phys. Rev. A 54, 1869 (1996).

[4] B. Schumacher and M. D. Westmoreland, Phys. Rev. A 56, 131 (1997); A. S. Holevo, IEEE Trans. Inf. Theory IT-44, 269 (1998).

[5] M. Fujiwara, M. Takeoka, J. Mizuno, M. Sasaki, Phys. Rev. Lett. 90, 167906 (2003); M. Takeoka, M Fujiwara, J. Mizuno, and M. Sasaki, Phys. Rev. A 69, 052329 (2004).

[6] S. Lloyd and S. L. Braunstein, Phys. Rev. Lett. 82, 1784 (1999).

[7] E. Knill, R. Laflamme, and G. J. Milburn, Nature 409, 46 (2001).

[8] R. Raussendorf and H. J. Briegel, Phys. Rev. Lett. 86, 5188 (2001).

[9] M. A. Nielsen, Phys. Rev. Lett. 93, 040503 (2004).

[10] D. E. Browne and T. Rudolph, LANL arXive quant-ph/0405157 (2004).

[11] S. J. Dolinar, Research Laboratory of Electronics, MIT, Quarterly Progress Report No. 111, 115 (1973).

[12] C. W. Helstrom, Quantum Detection and Estimation Theory (Academic Press, New York, 1976).

[13] R. S. Kennedy, Research Laboratory of Electronics, MIT, Quarterly Progress Report No. 108, 219 (1973).

[14] R. S. Bondurant, Opt. Lett. 18, 1896 (1993).

[15] M. Sasaki and O. Hirota, Phys. Lett. A 210, 21 (1996).

[16] M. Sasaki and O. Hirota, Phys. Rev. A 54, 2728 (1996).

[17] JM Geremia, LANL arXive quant-ph/0407205 (2004).

[18] A. S. Holevo, Sov. Math. 26, 3 (1982).

[19] K. Yamazaki, Quantum Aspects of Optical Communications, Vol. 378 of Springer Series of Lecture Notes in Physics (O. Hirota and S. Reynaud, eds., SpringerVerlag, Berlin, 1991), pp. 367-375.

[20] P. van Loock and N. Lütkenhaus, Phys. Rev. A 69, 012302 (2004).

[21] N. Lütkenhaus, J. Calsamiglia, and K.-A. Suominen, Phys. Rev. A 59, 3295 (1999).

[22] J. Calsamiglia and N. Lütkenhaus, Appl. Phys. B 72, 67 (2001). 
[23] S. J. van Enk, Phys. Rev. A 66, 042313 (2002).

[24] B. Huttner, N. Imoto, N. Gisin, and T. Mor, Phys. Rev. A 51, 1863 (1995).

[25] M. D. Srinivas and E. B. Davies, Opt. Acta 28, 981 (1981); M. D. Srinivas and E. B. Davies, ibid. 29, 235 (1982).

[26] M. Ueda, N. Imoto, T. Ogawa, Phys. Rev. A 41, 3891 (1990).

[27] M. Ban, Phys. Rev. A 49, 5078 (1994).

[28] C. W. Gardiner and P. Zoller, Quantum Noise (2nd ed.) (Springer-Verlag, Berlin, 2000).

[29] H. M. Wiseman and G. J. Milburn Phys. Rev. Lett. 70, 548 (1993).

[30] Regardless of the number of photodetections, the signal loses its energy during the measurement process. If the signal contains a vacuum-state component, a zero-photon counting event increases the probability amplitude for the vacuum [the second exponential term in Eq. [12]]. The average energy of the signal then decreases. Even if the initial signal does not contain the vacuum component, e.g. a single-photon state, the process in our setup still acts as a dissipative process. Since a displacement is not an energy preserving operation, it redistributes the amplitude coefficients of the partial state [the mode B in Fig. 2]a)] across the different number states including the vacuum state. As a result, even if the photon counting projects the partial state onto the vacuum, the partial state may have contained non-zero energy before the displacement. In other words, this event can create some vacuum-state component in the remaining signal [mathematically, this is due to the annihilation operator in the first exponential term of Eq. [12] ]. As mentioned above, once the state includes the vacuum component, the state loses its energy due to photon counting.

[31] P. van Loock, P. Raynal, and N. Lütkenhaus, in preparation.

[32] Since the average energy of each signal is finite and the displacement function in Eq. (28) is square integrable, $N_{\text {tot }}$ does not diverge even in the limit of $L \rightarrow \infty$.

[33] J. Calsamiglia, Phys. Rev. A 65, 030301(R) (2002). 